\title{
Extraction d'uranium et perpétuation du colonialisme interne au Nouveau-Mexique
}

Uranium extraction and perpetuation of internal colonialism in New Mexico

Extracción de uranio y perpetuación del colonialismo interno en Nuevo México

\section{Lucie Genay}

\section{OpenEdition}

\section{Journals}

\section{Édition électronique}

URL : https://journals.openedition.org/ideas/1734

DOI : $10.4000 /$ ideas. 1734

ISSN : 1950-5701

\section{Éditeur}

Institut des Amériques

\section{Référence électronique}

Lucie Genay, «Extraction d'uranium et perpétuation du colonialisme interne au Nouveau-Mexique », IdeAs [En ligne], 8| 2016, mis en ligne le 20 décembre 2016, consulté le 20 octobre 2022. URL : http:// journals.openedition.org/ideas/1734; DOI : https://doi.org/10.4000/ideas.1734

Ce document a été généré automatiquement le 20 octobre 2022.

\section{(i) $\odot$

Creative Commons - Attribution - Pas d'Utilisation Commerciale - Pas de Modification 4.0 International - CC BY-NC-ND 4.0

https://creativecommons.org/licenses/by-nc-nd/4.0/ 


\title{
Extraction d'uranium et perpétuation du colonialisme interne au Nouveau-Mexique
}

Uranium extraction and perpetuation of internal colonialism in New Mexico

Extracción de uranio y perpetuación del colonialismo interno en Nuevo México

\author{
Lucie Genay
}

\section{Introduction}

L'histoire moderne du Nouveau-Mexique, le berceau de l'ère nucléaire, est concomitante de l'émergence d'une Amérique nucléaire qui a fait de l'Ouest américain un territoire envahi par l'atome ${ }^{1}$. L'uranium, ou Leetso, qui signifie« poussière jaune »en Navajo, est devenu le minerai le plus important du mondeà la suite du Projet Manhattan ${ }^{2}$ (1942-1945). Il a bouleversé la vie sur le plateau du Colorado et continue de susciter la controverse dans les régions qui exploitent leurs gisements. Après que Paddy Martinez, un Indien Navajo néo-mexicain, eut découvert de l'uranium dans un morceau de roche calcaire Todilto àHaystack Butte sur les terres du Santa Fe Railroaden 1950, une croissance spectaculaire propulsa la petite ville néo-mexicaine de Grants, baptisée «capitale mondiale de la carotte» dans les années 1940, vers un nouveau statut de «capitale mondiale de l'uranium ». À l'instar de la ruée vers l'or de 1848-1853 en Californie, la découverte des gisements fit de la région un aimant pour les prospecteurs, les entreprises d'extraction et les travailleurs locaux,pour beaucoup des Indiens Navajo et Laguna,qui avaient particulièrement besoin d'un emploi. Cette analogie entre le passé pionnier, image caractéristique de la conquête de l'Ouest, et la ruée vers l'uranium illustre le caractère cyclique du colonialisme interne américain. Dans le cas de l'uranium néo-mexicain, on identifie deux grandes périodes dans ce cycle: celle du nucléaire principalement militaire, puis le basculement vers un nucléaire davantage civil. L'effacement du poids et du rôle des instances étatiques, qui entraina le premier déclin minier dans la région, est à la charnière entre ces deux 
époques. Pour autant, la renaissance nucléaire au début des années 2000 a fait de la production d'uranium aux États-Unis une question éminemment actuelle qui s'inscrit dans un débat mondial sur le nucléaire. Ainsipeut-on considérer que l'extraction de l'uranium serait le trait d'union entre le passé colonial de la région et les problématiques de son avenir au sein de l'industrie nucléaire. Cet article ${ }^{3}$ s'attache à démontrer en quoi l'histoire de l'uranium est symptomatique de la relation néocoloniale entre les régions pauvres et isolées de l'Ouest et le reste du pays. En outre, il s'agit d'interroger comment l'évolution de la situation post-mine, qui inclut lesimpacts environnementaux et sanitaires,a influencé les nouvelles prises de position par rapport à l'extractiond'uraniumau niveau local alors que les pressions économiques sont restées relativement similaires malgré le boom économique qu'a connu l'État après la Seconde Guerre mondiale.

\section{Les promesses de l'uranium : un autre cycle de conquête}

\section{Images et schémas de la conquête}

2 On trouve au Nouveau-Mexique une certaine tradition des industries extractives. Au tournant des $\mathrm{XIX}^{\mathrm{e}}$ et $\mathrm{XX}^{\mathrm{e}}$ siècles, de petites villes apparaissaient partout où des gisements de charbon, d'argent, d'or, de zinc, de cuivre, de potasse ou de pétrole étaient découverts. Silver City prit même le nom du métal qui l'avait créée. Le secteur minier, deuxième source de revenu de l'État après les activités agricoles, était un pilier de l'économie locale: d'abord l'or et l'argent, puis le cuivre et le charbon auxquels succéda le pétrole (McDonald B., 1988: 11). Certaines de ces communautés minières furent victimes par la suite des fluctuations économiques sur les marchés des minerais et devinrent des villes fantômes. Parce que la région était dépendante de cette production de matières premières pour des marchés extérieurs, Gerald Nash a qualifié l'Ouest américain de zone « sous-développée » et de «tiers-monde de l'Amérique ». Les États de l'Ouest étaient ainsi des colonies internes sous l'égide de l'Est industrialisé, principal acheteur de leurs produits (Nash G., $1990: 2)^{4}$.Selon Nash, la Deuxième Guerre mondiale et l'action du gouvernement fédéral aurait mis fin à ce colonialisme interne ; or, la bombe atomique et l'entrée dans la Guerre froide ont ouvert un nouveau marché minier qui évolua selon des contraintes très particulières mais non sans répéter certains schémas du passé. L'histoire de l'extraction de l'uranium au Nouveau-Mexique reflèteainsi à la fois l'héritage de la conquête de l'Ouest et sa perpétuation.

3 Les prospecteurs d'uranium des années 1950 n'étaient pas si différents des trappeurs, des chercheurs d'or ou des cowboys des générations précédentes. Des manuels de prospection leur étaient distribués par la Commission de l'énergie atomique (AEC) qui considérait qu'un programme national de production d'uranium était indispensable pour augmenter l'arsenal atomique et ces manuels servaient à ce que n'importe quel novice puisse «faire fortune» sans investissement majeur ou connaissance particulière.Les gisements découverts étaient principalement situés sur les terres du Santa Fe Railroad, mais aussi sur les propriétés privées de quelques ranchers, qui détenaient les droits d'exploitation des minerais, et sur le domaine public où les prospecteurs individuels durent se battre pour faire valoir leurs droits. Ainsi, des migrants d'autres États vinrent tenter leur chance; en mars 1955, Lewis Lothman, un 
Texan de Houston découvrit le gisement d'Ambrosia Lake où quatre usines furent construites. Kevin Fernlund écrit dans la New Mexico Historical Review qu'envisager cette ruée vers l'uranium à travers l'imaginaire « du old West offrait au pays un contraste familier, quoique mythique, à la réalité effrayante d'un monde au bord de la dévastation nucléaire» (Fernlund K., 1994: 350).Même si la comparaison, qui sembleincontournable dans un tel contexte, ne servait que d'image rassurante pour associer la matière première d'armes de destruction massive à l'or ou à l'argent des pionniers, cet élan vers l'uranium sponsorisé et contrôlé par le gouvernement n'en était pas moins un mouvement de conquête au même titre que l'avancée des précédentes vagues de migrants en quête de fortune. La différence majeure réside dans le rôle endossé par gouvernement fédéral par l'intermédiaire de l'AEC et dans des conséquences socio-environnementales sans précédent.

Peu après la découverte de Martinez, un pilote de l'Anaconda Copper Mining Company qui avait obtenu un permis de prospection détecta un immense gisement d'uranium sur la réserve du Pueblo de Laguna. L'entreprise dut passer un accord avec la tribu, qui lui loua plus de 3000 hectares et lui attribua des droit miniers en échange de redevances sur ses profits selon un schéma classique dans l'histoire des relations entre Indiens et pionniers américains. Cette fois, par contre, la défense nationale pesait aussi dans la balance. On faisait appel au patriotisme et à la loyauté des Indiens envers la patrie. En 1953, Anaconda signa un contrat avec l'AEC pour construire une usine de traitement à Bluewater pour l'uranium extrait de la mine ouverte baptisée Jackpile, à Laguna. Un article de presse de 1957, intitulé Uranium Discovery Makes Laguna Indians Modern 'Rags to Riches' Story ${ }^{5}$, met l'accent sur les nouveaux signes de confort moderne dus à la soudaine augmentation des revenus dans le village : électricité, antennes de télévision, postes de radio, véhiculesmotorisés, machines à laver et gazinières (Tucker B., 1957). Pendant que l'Amérique découvrait l'avenir très prometteur de l'atome, ce qui donnait lieu à des spéculations aussi mirobolantes que des voitures atomiquesou un climat plus doux grâce au bombardement atomique de la calotte polaire ${ }^{6}$,les promesses de l'uranium nourrissaient chez les résidents néo-mexicains l'espoir d'atteindre la prospérité économique à laquelle ils aspiraient depuis la Grande Dépression des années 1930.Le journaliste Wayne Winters écrivit en 1951 dans le New Mexico Magazine "L'uranium est arrivé à Grants! Et qui plus està point nommé car cette ville âgée de soixante-huit ans au nord-ouest du Nouveau-Mexique était dans une situation bientôt critique suite à l'épuisement de certaines de ses industries. [...] La population locale [...] déborde de joie.» (Winters W., 1951: 13) Les nouvelles opportunités d'emploi étaient présentées comme salvatrices pour une région isolée et épuisée. Ceci illustre le caractère cyclique des industries extractives qui évoluent au gré des valeurs attribuées aux ressources naturelles, une industrie en remplaçant parfois une autre dans les régions les plus pourvues.Au Mont Taylor ${ }^{7}$, la mine de pierres ponce, très active pendant la guerre, était sur le déclin et soixante-quinze familles avaient perdu leur gagne-pain après la fermeture du site forestier de la New Mexico Timber Company en 1951. Par conséquent, la population locale accueillit la découverte d'un immense gisement d'uraniumavec beaucoup d'enthousiasme.

\section{Promesses économiques, pauvreté et ignorance}

5 Le flot de personnes venant s'installer à Grants transforma radicalement la ville : la population de 2200 habitants en 1950 est passée à 10200 personnes dix ans plus tard. 
En 1981, un nouveau comté fut même créé ; il fut baptisé Cibola en référence au mythe des sept cités d'or qui avait attiré l'expédition menée par l'explorateur espagnol Francisco Vásquez Coronado en 1540, un clin d'œil à l'industrie minière devenu l'Eldorado nucléaire du pays. L'uranium s'est immiscé partout, y compris dans la culture locale avec l'ouverture d'un Uranium Café sur la mythique Route 66 et avec l'organisation d'un festival où l'on élisait le meilleur prospecteur de l'année et un concours de beauté où l'on élisait une Miss Energie Atomique qui se voyait offrir un chargement d'uranium en guise de prix. Le développement de la ville dépendait aussi du minerai par le biais des impôts locaux versés par les entreprises extractives, et des fonds fédéraux. En 1968, une nouvelle branche de l'Université deNew Mexico State fut fondée en coopération avec les écoles municipales de Grants pour former les étudiants au travail de la mine. En parallèle, les représentants politiques tels que le sénateur Clinton P. Anderson, fervent supporter de l'industrie nucléaire lorsqu'il était président du Comité mixte sur l'énergie atomique (1955-1961), jouèrent également un rôle dans la promotion de l'industrie etl'ouverture de nouvelles minesau Nouveau-Mexique pour booster l'économie locale. Précisons que le Nouveau-Mexique a irrémédiablement gardé sa place parmiles cinq États les plus pauvres du pays depuis son entrée dans l'Union en $1912^{8}$.

6 Le rapport entre le développement de l'uranium et le passé colonial du NouveauMexique ne se limite donc pas aux amalgames entre mythes fédérateurs et exploitation minière, ni à la répétition de certains schémas. Il s'agit également d'un lien entre angoisses économiques et promesses d'avenir qui pousse un État et sa population à approuver et promouvoir le développement d'une industrie risquée,même si l'ignorance du danger fait aussi pleinement partie de l'équation. En mars 1975, une étude du Comité à l'énergie nucléaire adressée au gouverneur du Nouveau-Mexique incitait l'État à participer davantage au cycle du combustible nucléaire car les avantages économiques seraient immenses d'après les auteurs, qui conseillaient d'encourager les industriels ou le gouvernement fédéral à construire une usine d'enrichissement dans l'État dès le début des années 1980 (Whan G., 1975 : 1-2)9 . Pour connaître le sentiment des Néo-Mexicains à l'égard d'une telle expansion, le Bureau d'étude de l'économie et des entreprises de l'Université du Nouveau-Mexique réalisa un sondage en Janvier 1977 ; celui-ci démontre que la majorité des participants était favorable à l'industrie du combustible nucléaire (45.9\%) et à son extension (46.5\%), pensant que les avantages supplanteraient les risques et les dangers environnementaux (48.5\%). La plupart était favorable à ce que les États-Unis utilisent plus d'énergie provenant du nucléaire (54\%) mais sans pour autant être prêts à approuver le stockage de déchets radioactifs dans leur État $(23.6 \%)^{10}$. L'intérêt de ce sondage est de révéler que même si la conclusion du rapport met en avant l'approbation des Néo-Mexicains du rôle de leur État dans l'industrie nucléaire, l'addition de toutes les autres réponses possibles (désintéressé, désapprouve, désapprouve fortement, incertain et ne dispose pas d'information suffisante) indique que la majorité penchait en réalité du côté de l'indécision, principalement liée à un manque de connaissances. Le rapport précise d'ailleurs que le nombre de personnes qui disaient ne pas disposer d'informations suffisantes pour répondre déclinait proportionnellement au niveau d'étude. Les plus diplômés avaient tendance à s'opposer à l'extension des installations nucléaires dans la région. Ce qui signifie que les personnes moins diplômées, probablement plus pauvres et originaires de la région, étaient prêtes à accepter plus facilement le danger et les risques à cause des pressions économiques (New Mexico Energy Institute, 1977: 2).Par la 
suite, le combat pour l'accès aux informations essentielles à la compréhension du facteur risque modifia radicalement l'éventail des opinions au Nouveau-Mexique.

7 Selon Richard White, les changements vers la modernité dans l'Ouest américain après la guerre «furent apportés d'une manière fortement influencée par l'histoire de l'Ouest" et ils "s'infiltrèrent par des chemins familiers" (White R., 1991: 461-462).Ainsi qu'on l'a fait remarquer ci-dessus, ces chemins familiers ont aussi tracé le développement de la capitale de l'uranium et de sa région. L'Ouest, terre d'accueil pour les aventuriers et les entrepreneurs, est entré dans un nouveau cycle avec l'ère nucléaire.Mais le système d'exploitation des ressources et de la main d'œuvre locale a maintenu le statut néocolonial des régions qui devinrent dépendantes de l'atome pour leur survie économique.

\section{Les effets d'un colonialisme économique}

\section{Le rôle du gouvernement et des grandes entreprises}

Les gigantesquesentreprises qui signèrent les contrats avec l'AECpour exploiter les gisements de la «Grants Uranium Belt » (Anaconda, Kerr-McGeeOil Industries, Phillips Petroleum Company et Vanadium Corporation of America) utilisèrent pour développer l'activité sur place des sous-traitants qui employèrent des mineurs néo-mexicains, pour beaucoup des Indiens Navajo et Laguna. Tous dépendaient de la demande d'uranium. À cause de cette dépendance, l'industrie devint une illustration ostensible de colonialisme économique.L'intensification de la production d'armes atomiques,et le fait d'avoir l'AEC pour unique acheteur, garantissait le succès des entreprises minières néomexicaines pendant la première phase de la Guerre froide.Ainsi l'extraction d'uranium s'ajouta aux autres facteurs de la forte croissance de l'après-guerre et fitde l'économie nucléaire du Nouveau-Mexique une industrie dite en anglais «du berceau au tombeau ", c'est-à-dire qui s'étend à toutes les étapes de la production(de la matière première jusqu'à la gestion du stock d'armes et des déchets en passant par la recherche et la production d'armes dans les laboratoires nationaux de Los Alamos et de Sandia à Albuquerque). Cependant, l'État devait la survie de son nouveau pilier économique aux évènements sur la scène internationale et se trouvait donc sous l'influence des décisions prises à Washington.

9 La première phase faste ne dura pas. L'évolution en dents de scie de l'extraction d'uranium entre 1950 et 1990 démontre les risques de cette situationà double tranchant où les entreprises d'extraction et de traitement, et, par conséquent, leurs ouvriers, étaient directement tributaires de la demande du gouvernement. Tant que celui-ci avait besoin d'uranium pour produire des ogives et des missiles nucléaires qui lui feraient gagner la course à l'armement avec les Soviétiques, le gouvernement soutenait la production nationale d'uranium grâce à des mesures protectionnistes. Quand le monopsone ${ }^{11}$ de "l'oncle Sam » établi par la loi de 1946 sur l'énergie atomique prit fin en 1970, les acheteurs privés ne purent compenser le départ de l'AEC sur un marché qu'elle avait elle-même créé. L'offre continua de grimper sans que la demande puisse absorber la production et les prix s'effondrèrent. Un regain d'activité apparut à la fin de la décennie quand le marché fut ouvert à d'autres acheteurs dans le domaine civil et la crise du pétrole stimula la demande pour d'autres sources d'énergie. En 1975, le Nouveau-Mexique produisait $40 \%$ de l'uranium nécessaire au nucléaire américain, 
selon le rapport du Comité à l'énergie nucléaire qui conseilla à l'État de « réévaluer » sa position par rapport aux besoins de la nation (Whan G., 1975 : 1).Mais cette deuxième phase de croissance ne dura pas non plus.L'industrie pâtit de l'ouverture du marché car la concurrence des producteurs étrangers, des Canadiens notamment, ébranla sérieusement l'industrie néo-mexicaine qui survivait grâce aux subventions. En janvier 1985 tous les sites d'exploitation avaient mis la clef sous la porte, ce qui laissa la région dans un état de désolation, parsemée de mines et d'usines abandonnées. Le chômage atteignit 30\%, la population de Grants passa de 20000 à 10000 personnes alors qu'il était prévu qu'elle atteigne 100000 personnes. En 1987, la Western Nuclear Incorporated de Jeffrey City dans le Wyoming intenta un procès au Département de l'énergie, l'accusant d'avoir tué le marché national de l'uranium en n'imposant aucune restriction sur l'enrichissement d'uranium étranger, ce qui violait la loi sur l'énergie atomique de 1954. Mais le tribunal déclara que des mesures protectionnistes n'auraient de toute façon pas permis de maintenir l'industrie à flot. Jeffrey City devint une ville fantôme. Grants échappa à ce sort. Un musée de l'extraction de l'uranium et la construction de trois prisons d'étatont assuré la survie de la ville. Michael Amundson, spécialiste de l'histoire des villes productrices d'uranium écrit: "Grants s'est volontairement enchaîné à l'industrie de l'uranium et au gouvernement fédéral par une sorte de colonialisme à la fois du gouvernement et des entreprises. » (Amundson M., 2002 : 93) Ce genre de colonialisme représente les liens entre économie locale et globale comme il en existe de nombreux exemples dans le monde (au Niger notamment en ce qui concerne l'uranium utilisé en France)où les entreprises et travailleurs locaux paient leur accèsà un marché plus grand de par leur soumission à des forces extérieures et incertaines.

\section{Quelle place pour les populations locales?}

La sociologue Valérie Kuletz écrit que les Indiens sont « ceux que l'on a rendu invisibles et qui continuent de l'être » alors que les acteurs du complexe nucléairesont « ceux qui choisissent d'être invisibles ». Ils évoluent dans une région que les premiers envisagent selon « une géographie de la terre sacrée et comme un lien [avec la terre] crucial pour leur survie culturelle " tandis que les seconds ont transformé cette région "en un paysage de sacrifice national. (Kuletz V., 1998: 7) L'uranium est une illustration particulièrement parlante de cette invisibilité, tout en renforçant le paradoxe entre exploitation et préservation qui divise ces communautés. Le Nouveau-Mexique compte vingt-deux nations indiennes qui représentent environ $10 \%$ de la population. Une grande partie du complexe militaro-industriel local a été construite à proximité de leurs réserves ou de leurs sites sacrés. Mais dans le cas de l'uranium, certaines tribus ont fait le choix d'accueillir l'activité sur leurs terres. Nombre d'Indiens sont allés travailler dans les mines et les usines et en ont tiré profit à court terme.Il s'avère néanmoins que les conditions de travail de ces ouvriers ne sont pas sans rappeler un système de travail de type colonial où les minorités sont considérées comme une réserve de main d'œuvre bon marché. Dans The Navajo People and Uranium Mining, les auteurs ont rassemblé les témoignages de mineurs Navajo. Parmi eux, George Tutt avait commencé à avoir des problèmes pulmonaires après plus d'une décennie à la mine où il avait travaillé en tant que "nettoyeur à la main ", c'est-à-dire qu'il enlevait les restes du minerai et les déchets à la pelle sans masque, ni gants,ni protection pour un salaire de $\$ 2.50$ de l'heure en 1960.Pour beaucoup de travailleurs, la mine fut le premier 
contact avec le salariat. Peu de mineurs connaissaientles mesures de sécurité. Floyd Frank, un autre mineur, s'est même demandé s'ils n'étaient pas considérés comme des Kleenex par le gouvernement. Tommy James, mineur à Shiprock, a décrit son travail comme de "l'esclavage » et insisté sur les différences entre les anglos qui avaient des cabanes avec douches et les Navajo qui vivaient dans leur tente. Il avait des maux de tête et des saignements de nez récurrents. Certains ouvriers se brûlaient avec des éclaboussures d'acide sulfurique parce qu'ils n'avaient pas d'équipement de protection. Les effluents coulaient de l'usine de traitement jusque dans la rivière. Enfin, Minnie Tsosie, la veuve d'un mineur, explique que ses deux filles ont subi une ablation de l'utérus. Les enfants et les femmes pouvaient en effet également être affectés en jouant dans la poussière radioactive, en lavant les habits ou en buvant de l'eau contaminée sur le site (Brugge D., Benally T. et Yazzie-Lewis E., 2006). L'entreprise Kerr-McGee, par exemple, qui employait environ soixante-dix Navajo et treize surveillants anglos, proposait des logements préfabriqués et des repas à ces derniers, tandis que les mineurs se débrouillaient. La Vanadium Corporation of America estimait que de telles installations, quand bien même elle les fournissait, ne seraient pas utilisées par les mineurs (Eichstaedt P., 1994 : 43).En plus des conditions de vie et de travail, les paies différaient d'environ $\$ 1$ de l'heure entre les travailleurs syndiqués et les Navajo (LaDuke W., 1979).

11 Les premiers effets sur la santé des employés sont apparus des années après l'ouverture des mines; principalement des affections des poumons (silicose, tuberculose, pneumonie, emphysème et cancers). En réalité, les premières études du service public américain de la santé datent des années 1950 mais elles ne concernaient que les mineurs blancs et aucune information n'était communiquée. En 1952, les travaux deWilliam Bale et John Harley sur les effets de l'énergie émise par les fil-isotopes du radon ont abouti à la définition de normes au travail mais il fut interdit aux scientifiques de publier leurs découvertes. À la fin de la décennie, les entreprises équipèrent leurs mines d'un système de ventilation. Cependant, beaucoup d'employés vivaient sur leur lieu de travail et étaient donc exposés de façon continue.

Toutefois, l'événement réellement "révélateur» de l'invisibilité historique de ces populations ne concerne pas les conditions de travail mais un accident. L'accidentradioactif le plus grave de l'histoire américaine.Le 16 juillet 1979, un barrage sur un bassin de stockage a cédé à l'usine de traitement d'uranium de la United Nuclear Corporation Church Rock, déversant mille tonnes de déchets radioactifs et 350 millions de litres d'eau contaminée vers un cours d'eau qui rejoint le Rio Puerco. Les particules contaminées ont été transportées en aval sur 130 kilomètres, passant devant les habitations d'environ 1700 Navajos. En dépit de sa gravité, la plupart des Américains n'ont jamais entendu parler de l'accident de Church Rock. Le contraste avec celui de Three Mile Island, qui avait libéré trois fois moins de radioactivité mais fait les gros titres de la presse nationale et internationale la même année, est frappant (Richards L., 2013). Pourtant, de nombreuses familles qui utilisaient la rivière quotidiennement et qui continuèrent ce jour-là à y abreuver leurs troupeaux ou à puiser de l'eau pour leurs besoins domestiques, furent directement exposées à des substances extrêmement dangereuses. L'absence de couverture ou même d'intérêt médiatique lorsque les personnes touchées font partie d'une minorité, dont l'influence politique est limitée, interpelle. Ce silence soulève des questions sur les formes de discrimination face à la gestion des risques mais il entretient aussi le voile d'ignorance parmi les populations 
concernées et rend les autres complices d'injustice environnementale. Dès la fin des années 1970, la mobilisation contre l'extraction d'uranium s'organisa chez les Navajo et les Pueblo au travers de rassemblements au Mont Taylor et autres sites importants de l'industrie, de conférences et de procès intentés contre les entreprises extractives et les agences gouvernementales.Dans la nébuleuse des révélations, des préoccupations environnementales et sanitaires et de la montée du militantisme se dessinèrent les nouvelles problématiques de la situation post-mine.

\section{Entre résidus toxiques, militantisme et nouvelles opportunités économiques}

\section{Décontamination : des négociations encore en cours}

13 La loi RECA (Radiation Exposure Compensation Act) de 1990 qui dédommagea les familles des vétérans atomiques et des downwinders (c'est-à-dire les populations contaminées par les retombées radioactives produites par les séries d'essais atmosphériques sur le site de tir du Nevada) promitégalement des compensations aux mineurs d'uranium sans englober pour autant toutes les maladies, tous les employés (ceux qui travaillaient dans les usines de traitement en étaient exclus) ou toutes les familles, car les épouses des mineurs Navajo rencontrèrent des difficultés pour prouver leur mariage. En 1994, 155 familles avaient reçu un chèque. De nombreux ouvrages sont consacrés aux impacts socio-environnementaux de ce qui a étédéfini comme un désastre technologiquepour les régions et les habitants, notammentpour la nation Navajo. On estime à un millier le nombre de mines abandonnées sur la réserve qui s'étend sur trois États (Arizona, Nouveau-Mexique et Utah) et qui a contribué à produire treize millions de tonnes de minerai. Depuis la désertion de la région par les entreprises, les résidus abandonnés continuent d'avoir un impact sur la santé des résidents.Le vent et l'eau dispersent les poussières radioactives, contaminant les végétaux, les sols et les animaux qui servent ensuite à nourrir la tribu. Les enfants jouent dans les mines.Les personnes et animaux égarés s'y abritent.Les matériaux de construction à base de terre contaminée servent à construire des routes et des habitations. Ainsi, onretrouve des particules radioactives dans les murs des hogans traditionnels. De plus, il est difficile d'expliquer aux résidentscomment fonctionne la radioactivité, phénomène invisible et inodore.Certains continuent donc de boire l'eau des sources ancestrales arguant que l'eau en bouteille n'a pas le même goût. Conséquence de ces expositions répétées, les statistiques montrent un taux de cancer élevé, ainsi qu'une forte occurrence de maladies rénales, d'hypertension et de problèmes pulmonaires parmi les Navajo ${ }^{12}$.

L'assainissement des sites abandonnésest donc devenuune priorité.La loi de 1978 (intitulée Uranium Mill Tailings Radiation Control Act) établit la liste des sites, nommés Title 1, éligibles pour une décontamination fédérale. Cette loi concernait exclusivement les sites où l'uranium était vendu à l'AEC avant 1971. Deux sites correspondaient à ce critère au Nouveau-Mexique: Ambrosia Lake et Shiprock. À l'usine Kerr-McGee de Shiprock où se trouve le plus grand amas de résidus dans le pays, on parle d'une mesa artificielle. Non sans ironie, les projets d'assainissement, pour la plupart encore en cours, ont régulièrement étél'occasion pour les résidents d'avoir accès à de nouveaux emplois. Entre 1985 et 1986, par exemple, le Département de l'énergie employa une quarantaine d'ouvriers Navajo pour consolider la montagne de résidus de Shiprock en 
la recouvrant d'une couche imperméable épaisse de deux mètres et d'une autre couche de roche pour que l'eau de pluie puisse ruisselerpar-dessus sans s'infiltrer. Malgré tout, les radiations ont contaminé la nappe phréatique.

15 Au Pueblo de Laguna, l'immense mine à ciel ouvert de Jackpile-Paguate, propriété d'Anaconda, est restée abandonnée pendant sept ans. Les citernes se remplissaient d'eau de pluie, invitant les enfants à venir nager et les animaux à s'y abreuver (Eichstaedt P., 1994 : 129 ; xvi). Les eaux de surface de la rivière Rio Paguate et du réservoir révélèrent la présence d'uranium isotopique (dont la structure a été modifiée). En 1986, après des années de négociations, le Pueblo de Laguna, le Bureau of Land Management, le Bureau des affaires indiennes et ARCO (l'entreprise qui a racheté Anaconda)ont passé un accord pour assainir la zone. Le projet mené par la tribu et financé par un fond de 45 millions de dollars versé par ARCO fut officiellement terminé en 1995 (Sittnick P., 1998). Néanmoins, les conclusions du dernier compte-rendu de septembre 2007 ont indiqué que le projet d'assainissement n'avait toujours pas répondu aux attentes en termes d'impact environnemental. L'Agence de protection environnementale (EPA) travaille actuellement à déterminer si la population est directement exposée à des contaminants. Aujourd'hui, trois des quinze sites néomexicains figurant sur la liste des priorités nationales, c'est-à-dire des sites dits super fund, sont d'anciennes mines ou usines de traitement : la Homestake Mining Company à Milan, la United Nuclear Corporation à Church Rock et la mine Jackpile-Paguate au Pueblo de Laguna (United States Environment Protection Agency).

16 En s'intéressant aux négociations menées et aux solutions proposées pour décontaminer les sites néo-mexicains, force est de constater qu'il demeure un flou scientifique et technique quant aux moyens de rétablir l'état originel de ces terres, ou du moins un état ne présentant pas de risque pour la santé. Le flou continue aujourd'hui malgré la multiplication des opinions d'experts employés par les entreprises, le gouvernement et les associations de militants. De la même façon, les négociations pour le financement de ces projets par les entreprises ou le gouvernement continuent, à l'image de l'accord conclu le 3 avril2014 qui impose à la Kerr-McGee Corporation de verser un milliard de dollars à la Nation Navajo pour l'assainissement de cinquante mines abandonnées sur leurs terres (Indian Country, 2014).Ainsi, la situation post-mine reste basée sur le fait que les résidents demeurent dépendants de l'argent versé par les mêmes acteurs de l'industrie qu'il y a cinquante ans.

\section{La renaissance nucléaire}

17 Au début des années 2000, la renaissance nucléaire qui promouvait l'énergie nucléaire comme une solution viable à l'épuisement des énergies fossiles a ranimé l'intérêt pour les gisements d'uranium américains dans le but d'augmenter l'autonomie énergétique du pays. C'était là l'une des priorités du partenariat international sur l'énergie nucléaire (Global Nuclear Energy Partnership, GNEP) de 2006 qui a pris fin avec l'arrivée de Barack Obama à la présidence. Les États-Unis ont cependant confirmé leur intérêt pour le nucléaire en adhérant au Cadre international de coopération sur l'énergie - International Framework for Nuclear Energy Cooperation (IFNEC) - et à la Commission Blue Ribbon pour l'avenir nucléaire de l'Amérique qui poursuit le débat sur la question des déchets produits par la filière.Pendant ce temps, les prix du minerai ont de nouveau augmenté et les industriels du secteur affirmaient en 2008 que la réouverture pourrait générer jusqu'à trente millions de dollars et près de 25000 
emplois dans la région de Grants. Une exagération d'après un rapport préparé pour le New Mexico Environmental Law Center qui conseillait de prendre en compte les coûts environnementaux et sociaux tout comme l'instabilité économique de l'activité (Power T. M., $2008: 1-2$ ).

De nouvelles entreprises, canadiennes pour la plupart, ont commencé à faire pression pour rouvrir les mines dans la zone au carrefour du Nouveau-Mexique, de l'Arizona, de l'Utah et du Colorado. Ainsi, la renaissance nucléaire serait en passe derelancer une troisième phase de croissance dans l'histoire de l'uranium américain. Cinq entreprises ont lancé des projets pour rouvrir les mines autour de Grants. Strathmore Minerals, reprise par Energy Fuels en 2013, avait fait la demande d'un permis en 2009 pour la mine de Roca Honda. On attend la réponse pour 2016. L'entreprise a aussi des projets à Marquez, Church Rock et Nose Rock. Après douze années de négociations, Hydro Resources Incorporated a reçu l'accord pour ses activités aux sites d'extraction par dissolution (ou lixiviation in situ, ISL) ${ }^{13}$ à Crownpoint et Church Rock. La Cotter Corporation a un projet au Mont Taylor et Laramide Resources Limited a un projet à La Jara Mesa (World Nuclear Association). La flamme extractive serait donc ranimée mais, contrairement aux précédentes vagues de pionniers, les derniers opportunistes du secteur minier doivent composer à présent avec la foule d'intervenants institutionnels et associatifs.

Tandis que certaines opérations de décontamination sont encore en cours, les représentants des tribus, les groupes de militants autochtones, et des associations mènent leur combat contre la reprise de l'activité et pour des solutions de décontamination plus efficaces. Les associations autochtones, comme la Eastern Navajo Diné Against Uranium Mining (ENDAUM), s'opposent catégoriquement à toute réouverture. L'association futd'ailleurs créée en 1994 lors de la proposition de Hydro Resources Incorporated de lancer un projet d'extraction par méthode ISL malgré les risques de pollution. Leona Morgan, coordinatrice d'ENDAUM, explique quelles techniques les industriels utilisent pour obtenir l'accès aux terres acquises par des membres de la tribu grâce au Dawes Act ${ }^{14}$. Les agents des entreprises d'extraction ciblent directement ces individus en difficulté financière, plutôt que les représentants de la tribu,pour leur faire des propositions attrayanteset ainsi obtenir les droits d'accès au terrain. C'est une tactique dite de "division et de conquête » d'aprèsENDAUM qui prévient que l'exploitation du site ISL ne durerait à nouveauqu'un temps.Cette étape serait suivie d'un travail de restauration auquel l'association ne croit pas car d'après Mitchell Capitan, résident de Crownpoint et fondateur d'ENDAUM qui a travaillé comme technicien des eaux pour la mine-test de MobilOil, l'extraction par dissolution ne présente pas de garantie quant au rétablissement des eaux souterraines à leur état de propreté initial. Point intéressant, plutôt que d'utiliser le mot "cycle" pour qualifier la production de combustible nucléaire, ENDAUM parle d'une "chaîne " à cause de l'absence de solutions pour traiter ou recycler les déchets produits par la filière, à l'inverse de ce qu'un cycle sous-entendrait (Morgan L., 2011). En effet, le cycle se trouve ailleurs, non pas dans la sphère technique mais plutôt dans l'histoire minière de l'Ouest américain.

20 La toile des acteurs dans le débat sur l'uranium est plus serrée que jamais et les enjeux sont de plus en plus élevés mais pour les populations locales, le dilemme reste le même entre la prise de risques et les pressions économiques. Ainsi, l'uranium est à la jonction entre un héritage toxique du passé et les ambitions du futur. Les entreprises qui 
vantent les vertus de méthodes d'extraction "propres" réutilisent toujours les mêmesincitations économiques pour étendre leurs projets dans la région tandis que les vestiges physiques des précédentes phases d'exploitation continuent d'impacter des résidents.

\section{Conclusion}

21 Le Nouveau-Mexique est un cas très particulier dans le complexe nucléaire américain. Berceau de l'ère atomique, il a accueilli avec enthousiasme le développement d'une industrie de pointe conditionnée par les circonstances de la Deuxième Guerre mondiale et de la Guerre froide. En redéfinissant son identité d'une région reculée, voire rejetée, de l'union en un fer de lance de la suprématie américaine, la «Terre de l'enchantement", ainsi qu'est surnommé l'État, a vécu une révolution économique phénoménale qui n'a pourtant pas permis de mettre fin à ses problèmes de pauvreté et de dépendance des forces économiques externes. L'histoire de l'uranium dans l'État s'intègre dans cette dynamique. Malgré les avantages indéniables de la croissance, le Nouveau-Mexique est non seulement resté extrêmement pauvre mais est également devenu de plus en plus inégalitaire. La situation du nord-ouest minier est symptomatique d'une région où l'histoire se répète, où les ressources et les habitants sont exploités avant d'être abandonnés par toute une industrie. Mais il ne s'agit pas de désigner les coupables et les victimes, car les discours sont multiples au sein de chaque groupe d'acteurs au niveau local. Seulement, aujourd'hui la problématique du nucléaire dépasse les frontières entre les États américains et les pays. À cause des temps de vie des éléments radioactifs qui se comptent en centaines de milliers d'années, les questions du nucléaire défient l'espace et le temps. C'est pourquoi la situation postmine appelle à des études transdisciplinaires et comparatives qui mettent en évidence les nouveaux schémas de cette nouvelle ruée vers l'or nucléaire.

\section{BIBLIOGRAPHIE}

Amundson, Michael A., Yellowcake Towns: Uranium Mining Communities in the American West, Mining in the American West, Boulder, CO, University Press of Colorado, 2002.

Begel, Deborah et David Lindblom. Dii'goToBaahane, Four Stories About Water. Produit par Deborah Begel et sponsorisé par Eastern Navajo Diné Against Uranium Mining, Sierra Club Environmental Justice Office, Southwest Research and Information Center, UNM Community Environmental Health Program et Connecting Higher Education Indigenously, 2012.

Boyer, Paul S., By the Bomb's Early Light: American Thought and Culture at the Dawn of the Atomic Age, Chapel Hill, NC, University of North Carolina Press, 1994.

Brugge, Doug, Timothy Benally et Esther Yazzie-Lewis (dir.), The Navajo People and Uranium Mining, Albuquerque, NM, University of New Mexico Press, 2006.

DeVoto, Bernard A., The Course of Empire, Boston, MA, Houghton Mifflin, 1952. 
Eichstaedt, Peter H., If You Poison Us: Uranium and Native Americans, Santa Fe, NM, Red Crane Books, 1994.

Fernlund, Kevin J., « Mining the Atom: The Cold War Comes to the Colorado Plateau, 1948-1958 », New Mexico Historical Review, Albuquerque, NM,vol. 69, n 4, octobre 1994, p. 345-356.

Genay, Lucie, « La conquête scientifique du Nouveau-Mexique: héritage local du Projet Manhattan ", thèse de doctorat en études anglophones, Université Grenoble Alpes, 2015.

Hevly, Bruce W. etJohn M. Findlay (dir.), The Atomic West, Seattle, WA, University of Washington Press, 1998.

Indian Country, « Navajo Nation to Get \$1 Billion in Historic Kerr-McGee \$5.15 Billion Cleanup Settlement », Indian Country, 4 avril 2014, http://indiancountrytodaymedianetwork.com/ 2014/04/04/navajo-nation-get-1-billion-historic-kerr-mcgee-515-billion-cleanupsettlement-154317, page consultée le 24 septembre 2015.

Kuletz, Valerie L., The Tainted Desert: Environmental Ruin in the American West, New York, NY, Routledge, 1998.

LaDuke Westigaard, Winona, « Uranium Mines on Native Land, The New Indian Wars », The Harvard Crimson, 2 mai 1979, http://www.thecrimson.com/article/1979/5/2/uranium-mines-onnative-land-pthe/?page=1, page consultée le 26 août 2015 .

McDonald, Brian, David Boldt et University of New Mexico, « The New Mexico Economy: History and Outlook », Albuquerque, NM, University of New Mexico, Institute for Applied Research, Bureau of Business and Economic Research, 1988.

Morgan, Leona, « ENDAUM Statement to the New Mexico Indian Affairs Committee », Gallup, NM, Eastern Navajo Diné Against Uranium Mining, 27 octobre 2011, http://www.nmlegis.gov/lcs/ handouts/IAC\%20110111\%20Statement\%20Leona\%20Morgan\%20ENDAUM.pdf, page consultée le 2 janvier 2015.

Nash, Gerald D., The American West Transformed: The Impact of the Second World War, Lincoln, NE, University of Nebraska Press, 1990.

New Mexico Energy Institute, « Attitudes of New Mexico Residents toward the Nuclear Fuel Industry », NMEI Report No. 76-513A, Albuquerque, NM, University of New Mexico, janvier 1977.

Power, Thomas Michael, « An Economic Evaluation of a Renewed Uranium Mining Boom in New Mexico », Santa Fe, NM, New Mexico Environmental Law Center, octobre 2008, http:// nmenvirolaw.org/images/pdf/nm-uranium-economics.pdf, page consultée le 19 avril 2015.

Richards, Linda M., « On Poisoned Ground », Chemical Heritage Magazine, Philadelphia, PA, Chemical Heritage Foundation, printemps 2013, https://www.chemheritage.org/distillations/ magazine/on-poisoned-ground, page consultée le 11 mars 2015.

Sittnick, Philip, « Uranium mining and its impact on Laguna Pueblo », juillet 1998, http:// miningwatch.ca/sites/default/files/umine.pdf, page consultée le 20 août 2015.

Tucker, Bob, « Uranium Discovery Makes Laguna Indians Modern ‘Rags to Riches' Story », 14 Juillet 1957, collection Governor John Dempsey Papers, 1936-1958, Santa Fe, NM: New Mexico State Records Center and Archive, Collection 1959-046, boîte 13191, G403, dossier 271, AEC Commission Subcommittee on Raw Materials Statements, Reports, $85^{\text {th }}$ Congress 1957.

United States Environment Protection Agency, « Search for Superfund Sites Where You Live »,United States Environmental Protection Agency, Washington, DC, https://www.epa.gov/ superfund/search-superfund-sites-where-you-live, page consultée le 11 décembre 2016. 
Whan, Glenn A., Chairman, « The State of New Mexico: Governor's Energy Task Force », Committee on Nuclear Energy, Santa Fe, NM, Executive Office of the Governor, 18 mars 1975.

White, Richard, "It's Your Misfortune and None of My Own » A History of the American West, Norman, OK, University of Oklahoma Press, 1991.

Winters, Wayne, « Uranium Boom at Grants »,New Mexico Magazine, Santa Fe, NM, mars 1951, p. $13-15 ; 52$.

World Nuclear Association, « U.S. Uranium Mining and Exploration »,World Nuclear Association, London, UK, http://www.world-nuclear.org/info/Country-Profiles/Countries-T-Z/Appendices/ US-Nuclear-Fuel-Cycle-Appendix-1--US-Uranium-Mining-and-Exploration-/, page consultée le 25 décembre 2014.

\section{NOTES}

1. Il s'agit principalement du nucléaire militaire (Hevly B., Findlay J., 1998).

2. Programme de recherche atomique américain qui a abouti à la création de la bombe atomique au laboratoire de Los Alamos, Nouveau-Mexique (Genay L., 2015).

3. Cet article s'appuie principalement sur les recherches que j'ai menées dans le cadre de mon doctorat sur l'impact de l'industrie nucléaire au Nouveau-Mexique.

4. Cette idée se rapproche également du concept proposé par Bernard DeVoto d'un «empire intérieur » aux États-Unis (DeVoto, B., 1952 : xxxi).

5. Traduction: Découverte d'un gisement d'uranium : une version moderne de l'histoire du pauvre qui devient riche pour les Indiens Laguna.

6. Ce que Paul Boyer nomme «l'utopie nucléaire » (Boyer, P., 1994 : 114).

7. Le Mont Taylor est aussi connu sous le nom de Turquoise Mountain (Tsoodzil) et fait partie des quatre montagnes sacrées qui marquent les points cardinaux pour la Nation Navajo. C'est aussi un lieu de pèlerinage pour les Indiens Pueblo.

8. Il était deuxième derrière le Mississippi en 2011 selon le Bureau du recensement des ÉtatsUnis.

9. Une telle usine appelée URENCO et gérée par Louisiana Energy Services existe depuis juin 2010 à Eunice au Nouveau-Mexique.

10. Ce qui sera finalement le cas avec l'ouverture du Waste Isolation Pilot Plant de Carlsbad en 1999.

11. Jusqu'en 1970, le gouvernement était l'unique acheteur légal de l'uranium produit aux ÉtatsUnis. Ce système garantissait aussi les prix pour encourager des entreprises privées à prospecter et extraire le minerai, un système gagnant-gagnant puisque le gouvernement assurait aux entreprises un marché et les entreprises servaient exclusivement les besoins du gouvernement. Un monopsone, à l'inverse d'un monopole où il n'y a qu'un seul vendeur, correspond à cette situation instaurée par la loi sur l'énergie atomique de 1946. L'AEC avait également le monopole des services d'enrichissement de l'uranium. Les centrales nucléaires durent ainsi vendre leur énergie à l'AEC.

12. Cf. le documentaire Dii'goToBaahane, Four Stories About Water, realisé en 2012 par Deborah Begelet David Lindblom, produit par Deborah Begel et sponsorisé par Eastern Navajo Diné Against Uranium Mining, Sierra Club Environmental Justice Office, Southwest Research and Information Center, UNM Community Environmental Health Program et Connecting Higher Education Indigenously. Ce documentaire a été diffusé dans plusieurs festivals de films sur l'uranium et à UNM. 
13. Il s'agit d'une technique d'extraction par la dissolution de l'uranium dans une solution liquide qui est ensuite pompée à la surface. Il y a donc peut l'impact à la surface et peu de résidus mais cela présente un risque de contamination des eaux souterraines. On connait aussi cette méthode sous le nom de récupération In Situ (ISR).

14. Cette loi de 1887 permit la division des terres tribales en petites parcelles privées que le président américain octroyait individuellement aux membres des tribus indiennes tout en leur accordant la citoyenneté américaine pour aider leur intégration dans la société.

\section{RÉSUMÉS}

L'histoire de l'uranium au Nouveau-Mexique commence dansles années 1950. Suite à la découverte d'un immense gisement à proximité de la petite ville de Grants, dans le nord-ouest, l'économie locale fut radicalement transformée par l'arrivée des prospecteurs et des entreprises extractives. Les hauts et les bas de l'industrie minière pendant et depuis la Guerre froide ont profondément marqué le paysage et les populations locales qui cherchent encore aujourd'hui à atténuer les stigmates du passé tout en répondant aux préoccupations économiques pour l'avenir. Cet article interroge le caractère cyclique de l'exploitation minière dans un État parmi les plus pauvres de l'Ouest américain. Il s'agit d'examiner la transition entre la ruée vers une ressource naturelle et la situation qui découle de son exploitation dans le contexte particulier du nucléaire. Les enjeux économiques et socio-environnementaux de ces dernières années, ainsi que le nouveau rôle des populations locales (notamment les indiens Navajo et Laguna en tant que nations) à travers un militantisme fort mais décousu, distinguent cette industrie des autres vagues migratoires liées au développement d'un secteur minier. Toutefois, certains aspects néocoloniaux laissent à croire que, si les enjeux ont changé, certains schémas sont restés les mêmes et perpétuent un héritage de dépendance et d'inégalité.

The history of uranium in New Mexico started in the 1950s. Following the discovery of a large deposit near the small town of Grants in the north-western part of the state, the local economy was dramatically transformed by the arrival of prospectors and extractive companies. The ups and downs of the mining industry during and after the Cold War have profoundly impacted the landscape and the local population who is still trying to alleviate the effects of the past while addressing the economic concerns of the future. This article focuses on the cyclic aspect of mining in one of the poorest states of the American West.It raises questionsas to the transition from the rush for natural resources to its post-exploitation situation in the particular context of the nuclear industry. The economic and socio-environmental issues of the last few years, as well as the new role played by the local population (Navajo and Laguna Nations in particular) through their strong but disconnected activism, set this industry apart from previous migratory waves connected to the development of one mining sector. Nonetheless, certain neocolonial characteristics show that, despite these new issues, some patterns have remained the same and perpetuate a legacy of dependence and inequality.

La historia del uranio en Nuevo-México comienza en los años 50. A raíz del descubrimiento de un inmenso yacimiento cerca de la ciudad de Grants, en el noroeste del Estado, la economía local se vio radicalmente transformada por la llegada de prospectores y de empresas de extracción.Los altibajos de la industria minera durante y desde el fin de la guerra fría han marcado 
profundamente el paisaje y las poblaciones locales, que hasta el día de hoy buscan atenuar las cicatrices del pasado, así como responder a las preocupaciones económicas del futuro. Este artículo analiza el carácter cíclico de la explotación minera en uno de los Estados más pobres del oeste de los Estados Unidos.Se interroga sobre la transición entre la explotación febril de los recursos naturales y la situación post-explotación en el particular contexto de un programa nuclear.Los desafíos económicos, sociales y medioambientales de los últimos años, así como el rol cada vez más preponderante de las poblaciones locales (las Naciones Navajo y Laguna principalmente) a través de un activismofuerte aunque desarticulado, distinguen a esta industria de otras olas migratorias vinculadas con el desarrollo de un sector minero. No obstante, ciertos aspectos neocoloniales hacen pensar que, a pesar de estos nuevos desafíos, algunos mecanismos permanecen intactos y perpetúan un legado de dependencia y de disparidad.

INDEX

Mots-clés : uranium, Nouveau-Mexique, colonialisme, nucléaire, Indiens

Keywords : uranium, New Mexico, colonialism, nuclear industry, Native Americans

Palabras claves : uranio, Nuevo México, colonialismo, industria nuclear, indios americanos

\section{AUTEUR}

\section{LUCIE GENAY}

Lucie Genay est maître de Conférences dans le département des études anglophones de l'Université de Limoges, Laboratoire Espaces Humains et Interactions Culturelles (EHIC) Lucie.Genay@unilim.fr 